\title{
Development of a micro swimming robot using optimised giant magnetostrictive thin films
}

doi:10.1533/abbi.2006.0025

Y. Zhang ${ }^{1,2}$, G. Liu² and H. Li ${ }^{1}$

${ }^{l}$ Key Laboratory of Dalian University of Technology Precision $\mathbb{E}$ Non-traditional Machining of the Ministry of Education, Dalian 116024, China

${ }^{2}$ Department of Aerospace Engineering, Ryerson University, 350 Victoria Street, Toronto, Ontario, Canada M5B 2 K3

\begin{abstract}
A fish-like swimming micro robot is developed using an optimised fin actuator made of giant magnetostrictive films (GMFs). The force oscillation dynamic model of a GMF cantilever with variable cross-section area is derived, and the propulsive model of the fish robot in liquid is established. A discrete variate method for optimising caudal fin configuration is proposed to optimise its propulsive force and drive efficiency under the constraints of fixed surface area and sufficient fin end strength. Both theoretical analysis and experimental results have confirmed that the optimised caudal fin configuration can generate more powerful propulsion and improved efficiency.
\end{abstract}

Key words: Swimming robot, giant magnetostrictive film, fin optimisation.

\section{INTRODUCTION}

Propelled by transverse body distortion, fish possess remarkable swimming capacities in water. Propulsive modes of fish are associated with high efficiency, excellent manoeuvrability and large acceleration (Webb and Weihs 1983).

The compound motion of caudal fin comprises oscillatory and translational motions, and the oscillatory motion generates more than $90 \%$ of the overall propulsive force (Sfakiotakis et al. 1999). A structural diagram of caudal fin is shown in Figure 1. The magnitude of propulsive force mainly depends upon its shape parameters such as the aspect ratio $K\left(K=B^{2} / s_{\mathrm{c}}\right.$, where $B$ is the span, $s_{\mathrm{c}}$ is the surface area), sweepback angle $\beta$, etc. Within a certain range, a larger aspect ratio leads to less friction and higher propulsion force and drive efficiency.

Curvature of the leading edge is also a crucial factor. Incurvate leading edge is propitious to reduce suction and avoid the separation of boundary layer, resulting in increased propulsive force (Breder 1926).

Higher fin rigidity generates larger propulsion force, but lower propulsive efficiency.

Corresponding Author:

G. Liu

Department of Aerospace Engineering

Ryerson University

350 Victoria Street, Toronto

Ontario, Canada M5B 2K3

Tel: (416) 979-5000 ext.7648; Fax: (416) 979-5056

Email: gjliu@ryerson.ca
In this article, a fish-like micro swimming robot is developed using an actuator made of thin giant magnetostrictive films (GMFs) to imitate oscillatory motion of caudal fin. When exited by an alternating magnetic field, the GMF fin vibrates similarly to a caudal fin of fish to generate propulsion from the interaction between the vibrating fin and the liquid.

In order to increase the propulsion force and drive efficiency of the proposed biomimetic micro swimming robot, shape optimisation of the GMF caudal fin is conducted. Although a larger aspect ratio generates more powerful propulsion under the constraint of a certain caudal fin area, it weakens the rigidity of fin end and decreases its drive torque and deflection amplitude of the GMF, resulting in less propulsion force. Design optimisation of the fin shape is required to maximize the propulsion force and efficiency.

In this article, a force oscillation dynamic model of GMF cantilever with variable cross-section area is established, and a propulsive model of micro swimming robot in liquid is derived. On the basis of the developed models, a discrete variate method is proposed for optimising caudal fin configuration under the constraints of constant surface area and sufficient fin end strength. Simulation and experimental results have confirmed the significant improvement of the optimised GMF caudal fin in terms of propulsion and drive efficiency.

\section{GMF CAUDAL FIN}

The GMF caudal fin is double-faced and made of magnetostrictive amorphous $\mathrm{T}_{\mathrm{b} 0.28} \mathrm{D}_{\mathrm{y} 0.72} \mathrm{~F}_{\mathrm{e} 1.93}$ and $\mathrm{S}_{\mathrm{m}} \mathrm{F}_{\mathrm{e} 1.95}$ thin 


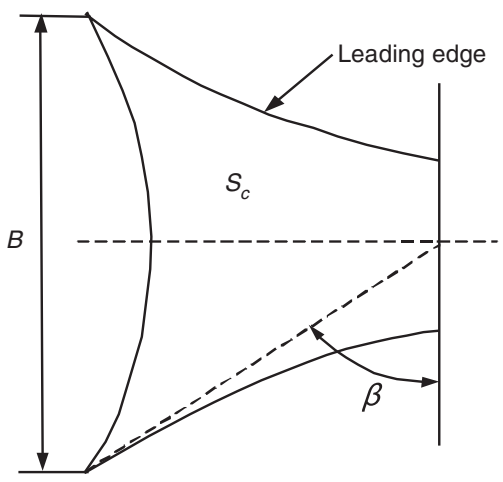

Figure 1 Configuration of caudal fin.

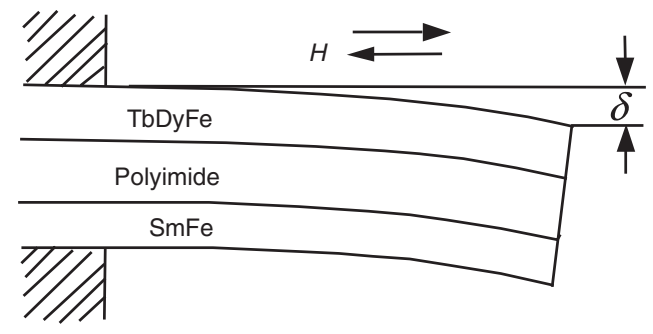

Figure 2 Deflection of a GMF fin.

films, sputtered on the opposite sides of a layer of polyimide film substrate with a thickness of $35 \mu \mathrm{m}$.

When a magnetic field is applied along the longitudinal direction (along the pipe axis), $\mathrm{T}_{\mathrm{b} 0.28} \mathrm{D}_{\mathrm{y} 0.72} \mathrm{~F}_{\mathrm{e} 1.93}$ film, a positive magnetostrictive material, expands and $S_{\mathrm{m}} \mathrm{F}_{\mathrm{e} 1.95}$ film, a negative magnetostrictive material, contracts. As a result, the fin deflects downwards. When an alternating magnetic field with variable frequency is applied along the longitudinal direction, the GMF fin vibrates in response to the alternating magnetic field, and propulsive force is generated by the interaction between the vibrating fin and the liquid. Cross-sectional diagram of a GMF fin is shown in Figure 2, where $H$ is the external alternating magnetic field applied to the fin, and $\delta$ denotes deflection of the free end of the GMF fin.

Theoretical analysis and experiments have shown that GMF fin in mode shape of the second-stage resonance frequency generates the most powerful propulsion. At the second-stage resonance frequency, vibration amplitude of a GMF fin reaches the highest value at the free end. Therefore, under the constraint of constant overall caudal fin area, relatively large free end is propitious to the increase of propulsion force, whereas the area size near the fixed end has little influence on propulsion. It is therefore predictable that the optimal configuration of a caudal fin features a large free end, similar to the shape of a caudal fish fin. Provided sufficient physical strength of a caudal fin, increasing its aspect ratio and sweepback angle can generate more powerful propulsion.

For design optimisation, it is necessary to describe the shape of GMF caudal fin mathematically. The upper leading edge of a caudal fin, as shown in Figure 3, could be

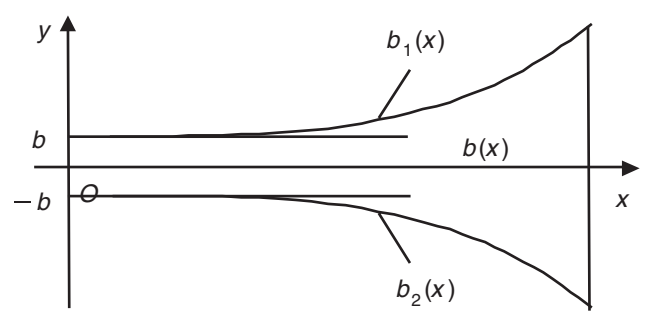

Figure 3 Configuration of a caudal fin.

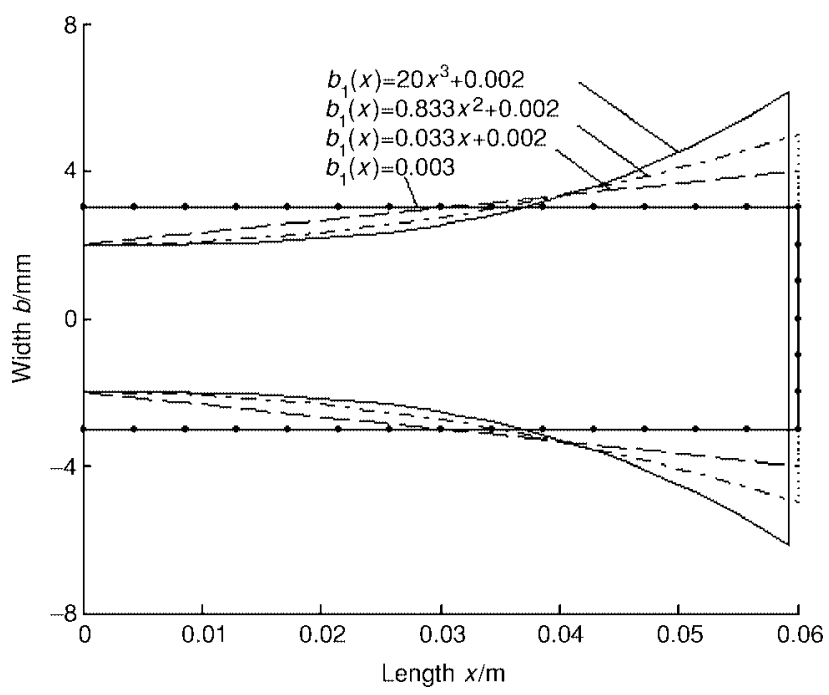

Figure 4 Various configurations of caudal fin.

described by a polynomial

$$
b_{1}(x)=a_{n} x^{c}+a_{n-1} x^{c-1}+\cdots+a_{1} x+b_{0},
$$

where $c$ is a positive integer. Generally, all the coefficients may or may not be zero. However, as the objective is to maximize the aspect ratio for a given overall fin area, we suppose that only the coefficient for highest power term is not zero, that is,

$$
b_{1}(x)=a x^{c}+b,
$$

where the coefficients $a$ and $b$ are positive. The value of $b$ is determined by the width of the fixed end of the caudal fin. This significantly simplifies the optimisation problem under consideration.

As shown in Figure 3, where the lower leading edge $b_{2}(x)$ is symmetric to $b_{1}(x)$ with respect to the axis $x$. Hence, the total width of the caudal fin is $b(x)=2 b_{1}(x)$. The total length of the caudal fin is $l$.

The task is now to determine the fin parameters $a, b, c$ and $l$ to generate the most powerful propulsion and highest efficiency, under the constraint of constant surface area of the caudal fin.

Four representative caudal fin configurations with the same fin area are shown in Figure 4, and their parameters are given in Table 1 . 
Table 1 Parameters of a caudal fin

\begin{tabular}{lllll}
\hline $\begin{array}{l}\text { Configuration of } \\
\text { caudal fin }\end{array}$ & $\begin{array}{l}\text { Chord length } l \\
(\mathrm{~mm})\end{array}$ & $\begin{array}{l}\text { Span } b \\
(\mathrm{~mm})\end{array}$ & $\begin{array}{l}\text { Span } \\
\text { ratio } K\end{array}$ & $\begin{array}{l}\text { Sweep angle } \\
\beta(\mathrm{rad})\end{array}$ \\
\hline$b_{1}(x)=0.033 x+$ & 60 & 8 & 0.178 & 0.067 \\
0.002 & & 10 & 0.278 & 0.083 \\
$b_{1}(x)=0.833 x^{2}+$ & 60 & 12.2 & 0.420 & 0.104 \\
0.002 & & & & \\
$b_{1}(x)=20 x^{3}+0.002$ & 59.2 & &
\end{tabular}

\section{DYNAMIC MODEL}

\section{Equivalent drive moment of GMFs}

The dynamic relationship between the magnetic field and magnetostrictive coefficient $\lambda$ is determined by interpolating experimental curves (Body et al. 1997).

$$
\lambda(H)=L\left[1-\exp \left(-e H^{f}\right)\right]+g H,
$$

where the parameters $L, e, f$ and $g$ are optimised to fit experimental data. This formula is used to calculate an internal magnetostrictive stress in the film, depending on the magnetic field. For a membrane, the even stress $\sigma_{\mathrm{m}}$ is (Honda et al. 1997)

$$
\sigma_{\mathrm{m}}= \pm \lambda E_{1} /\left(1+\mu_{\mathrm{p}}\right)
$$

where $E_{1}$ is the Young's modulus of GMF; $\mu_{\mathrm{p}}$, Poisson's coefficient of GMF.

The minus sign in Equation (2) represents compressive stress inside the $\mathrm{T}_{\mathrm{b} 0.28} \mathrm{D}_{\mathrm{y} 0.72} \mathrm{~F}_{\mathrm{e} 1.93}$ thin layer when it extends, and the positive sign in Equation (2) means tensile stress inside the $\mathrm{S}_{\mathrm{m}} \mathrm{F}_{\mathrm{e} 1.95}$ thin layer when it contracts under the application of magnetic field along the longitudinal direction.

A classical bending theory for trimorph cantilever is applicable to the magnetostrictive thin-film cantilever, as shown in Figure 5(a), for obtaining its disturbing drive moment by stress analysis inside a thin-film cantilever.

Assuming that a neutral layer is a section that overpasses axis $x$. The neutral axis can be obtained as follows (Quant and Seemann 1995):

$$
\int_{(n-1) h}^{n h} E(z) z \mathrm{~d} z=0
$$

where $z$ is the coordinate along the axis; $E(z)$, Young's modulus as a function of thickness; $E_{1}$, Young's modulus of GMF; and $E_{2}$, Young's modulus of substrate.

As indicated in Figure 5, $h$ is the thickness of the cantilever, the upper and the lower surfaces are located at $z=n h$ and $z=(n-1) h$, respectively $(0<n<1)$. Stress distribution of the cantilever in a section $a-a^{\prime}$ is shown in Figure 5(b) when it bends. Compressive stress $\sigma_{\mathrm{m}}$ of $\mathrm{T}_{\mathrm{b} 0.28} \mathrm{D}_{\mathrm{y} 0.72} \mathrm{~F}_{\mathrm{e} 1.93}$ can be regarded as constant when it expands, because the thickness of a sputtered film is quite thin, and tensile stress $-\sigma_{m}$ of $\mathrm{S}_{\mathrm{m}} \mathrm{F}_{\mathrm{e} 1.95}$ can also be regarded as constant when it contracts for the same reason.
Internal stress is tensile above the neutral layer, whereas internal stress below the neutral layer is compressive. The disturbing drive moment of trimorph cantilever is described as follows:

$$
M(x, t)=\int_{-0.5 h}^{0.5 h} E(z) \lambda(z) z B(x) \mathrm{d} z,
$$

where $B(x)$ is the width of cantilever; $\lambda(z)$, strain as a function of thickness.

\section{Dynamic model of force oscillation}

For a GMF cantilever with variable cross-section area and a total length $l$, assuming the mass per unit length is $\rho(x)$, stiffness $k(x)$ in section plane is also a function of $x$ and $k(x)=E I(x)$. A typical differential equation for describing a beam in free vibration without any damping coefficient and alternating load can be written as (Tse et al. 1978)

$$
\frac{\partial^{2}}{\partial x^{2}}\left[k(x) \frac{\partial^{2} z}{\partial x^{2}}(x, t)\right]+\rho(x) \frac{\partial^{2} z}{\partial t^{2}}(x, t)=0 .
$$

The solution of the above equation is the vibration deflection of GMF beam along its full length, which can be described in the form of infinite series about its eigenfunctions as follows:
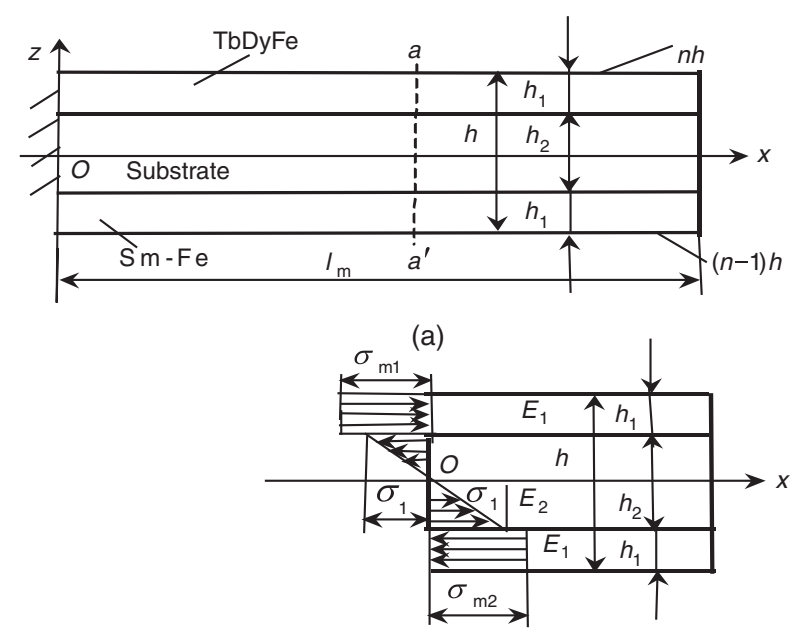

(b)

Figure 5 Stress analysis of a GMF and its equivalent moment. 


$$
z(x, t)=\sum_{i=1}^{\infty} V_{i}(x) q_{i}(t),
$$

where $q_{\mathrm{i}}(t)$ is the generalized coordinate; $V_{\mathrm{i}}(x)$, eigenfunction.

Using Lagrange equation and the theory of virtual work, a typical differential equation of force oscillation in terms of generalized coordinates $q_{i}(t)$ driven by a disturbing moment can be described by

$$
\mathbf{M} \ddot{q}_{i}(t)+\mathbf{C} \dot{q}_{i}(t)+\mathbf{K} q_{i}(t)=Q_{i}(t),
$$

where

$$
\begin{aligned}
\mathbf{M} & =\left[\begin{array}{ccc}
m_{1} & \cdots & 0 \\
\vdots & & \vdots \\
0 & \cdots & m_{n}
\end{array}\right] \quad \mathbf{C}=\left[\begin{array}{ccc}
c_{1} & \cdots & 0 \\
\vdots & & \vdots \\
0 & \cdots & c_{n}
\end{array}\right] \\
\mathbf{K} & =\left[\begin{array}{ccc}
k_{1} & \cdots & 0 \\
\vdots & & \vdots \\
0 & \cdots & k_{n}
\end{array}\right] \\
m_{i} & =\int_{0}^{l} \rho(x) V_{i}^{2}(x) \mathrm{d} x \\
c_{i} & =\int_{0}^{l} r(x) V_{i}^{2}(x) \mathrm{d} x \\
k_{i} & =\int_{0}^{l} k(x)\left[V_{i}^{\prime \prime}(x)\right]^{2} \mathrm{~d} x \\
Q_{i}(t) & =\int_{0}^{l} M(x, t) V_{i}^{\prime}(x) \mathrm{d} x
\end{aligned}
$$

where $V_{i}^{\prime}(x)$ is the first-order derivative eigenfunction; $V_{i}^{\prime \prime}(x)$, second-order derivative eigenfunction.

Steady-state solution of force oscillation in terms of generalized coordinates $q_{i}(t)$ is

$$
q_{i}(t)=\phi \sin (\omega t-\varphi)+\frac{Q_{i}(t)}{f_{i}^{2}},
$$

where

$$
\begin{aligned}
\phi & =\frac{Q_{i}(t)}{\sqrt{\left(f_{i}^{2}-\omega^{2}\right)^{2}+\left(2 \zeta f_{i} \omega\right)^{2}}} ; \\
\varphi & =\arctan \left(\frac{2 \zeta_{i} f_{i} \omega}{\left(f_{i}^{2}-\omega^{2}\right)}\right) ;
\end{aligned}
$$

$\zeta_{i}$, damping coefficient.

\section{Deflection of a GMF caudal fin}

In order to conform $z(x, t)$, the vibration deflection of GMF fin in variable section area, according to Equation (5), the eigenfunctions $V_{i}(x)$ have to be solved first, as the steady-state deflection of GMF itself in terms of generalized coordinates $q_{i}(t)$ also involves the eigenfunctions $V_{i}(x)$.
It is difficult to obtain the analytic solutions of natural frequencies and the eigenfunctions $V_{i}(x)$ of a GMF fin with variable cross-sectional area. However, the Rayleigh and Ritz method is applied successfully to obtain their numerical solutions.

Based on an assumption that the eigenfunctions $V_{i}(x)$ corresponding to resonance frequency $f_{i}(i=1,2,3)$ in terms of series is as follows:

$$
V_{i}(x)=\sum_{j=1}^{n} a_{j} u_{j}(x),
$$

where $a_{j}$ is undetermined coefficient; $U_{j}(x)$, non-linear correlated function.

Characteristic values can be derived from systematical characteristic equation according to the Rayleigh and Ritz method.

$$
\mathbf{k}^{\prime} a=\omega^{2} \mathbf{m}^{\prime} a,
$$

where $\mathbf{k}^{\prime}, \mathbf{m}^{\prime}$ are $n \times n$ symmetrical and constant matrices; $\omega$, natural frequency of GMF fin.

$$
\begin{aligned}
k_{i j}^{\prime} & =\int_{0}^{l} E I(x) u_{i}^{\prime \prime}(x) u_{j}^{\prime \prime}(x) \mathrm{d} x \\
m_{i j}^{\prime} & =\int_{0}^{l} \rho(x) u_{i}(x) u_{j}(x) \mathrm{d} x \\
i, j & =1,2, \ldots, n \\
a^{T} & =\left\{a_{1}, a_{2}, \ldots a_{n}\right\}
\end{aligned}
$$

where $u_{i}^{\prime \prime}(x)$ is the second-order derivative functions in non-linear correlation under boundary conditions.

Consider the boundary conditions of the fixed end of cantilever. When $x=0$, both displacement and deflection angle at the fixed end are zero. Therefore, the nonlinear correlated functions under the boundary conditions can be simply written as $u_{j}(x)=x^{j+1}$. The formulated eigenfunction $V_{i}(x)$ is more accurate for higher values of $n$, but it results in complicated calculation. Usually, $n=$ 6 is enough to guarantee the accuracy in practical problems. Therefore, $u_{j}(x)=x^{j+1}(j=1,2, \ldots, 6)$. For a caudal fin with variable cross-section, the three lower order natural frequencies, $f_{i}(i=1,2,3)$, and the three corresponding normalized eigenfunctions, $V_{i}(x)(i=1,2,3)$, can be formulated. By introducing the three normalized eigenfunctions $V_{i}(x)$ into Equations (5) and (7), the deflection in steady state $z(x, t)$ of the GMF fin can be obtained.

Let us take the three caudal fins with different configurations in Figure 4 as examples to show the calculation procedure. From Equations (8) and (9), the calculated results of their first three-stage resonance frequencies, $f_{i}(i=1,2,3)$, and their corresponding normalized eigenfunctions, $V_{i}(x)$, are shown in the following:

(1) $y=0.0333 x+0.002 l=0.06$

$$
f=[11.7,81.3,234.1]
$$




$$
\begin{aligned}
a^{(1)}= & {[248476.4,-3252515,25464255,-0.1890} \\
& \times 251 \times 10^{9}, 0.1375924 \times 10^{10},-0.4426281 \\
& \left.\times 10^{10}\right] \\
a^{(2)}= & {\left[1658146,-0.5462288 \times 10^{8}, 0.1945167\right.} \\
& \times 10^{9}, 0.8402133 \times 10^{10},-0.9093011 \\
& \left.\times 10^{11}, 0.2140364 \times 10^{12}\right] \\
a^{(3)}= & {\left[-4837322,0.2510007 \times 10^{9}, 0.1671062\right.} \\
& \times 10^{8},-0.1831 \times 10^{12}, 0.3313213 \times 10^{13}, \\
& \left.-0.1773199 \times 10^{14}\right]
\end{aligned}
$$

(2)

$$
\begin{aligned}
y= & 0.8333 x^{2}+0.002 l=0.06 \\
f= & {[11.7,78.9,230.9] } \\
a^{(1)}= & {[222248.5,-1598498,-0.2230771} \\
& \times 10^{8}, 0.5168418 \times 10^{9},-0.3837539 \\
& \left.\times 10^{10}, 0.1083383 \times 10^{11}\right] \\
a^{(2)}= & {\left[1586078,-0.4144731 \times 10^{8},-0.5246221\right.} \\
& \times 10^{9}, 0.2581622 \times 10^{11},-0.286549 \\
& \left.\times 10^{12}, 0.1050199 \times 10^{13}\right] \\
a^{(3)}= & {\left[-4950851,0.2549185 \times 10^{9}, 0.2681288\right.} \\
& \times 10^{9},-0.198257 \times 10^{12}, 0.3579971 \\
& \left.\times 10^{13},-0.1927949 \times 10^{14}\right]
\end{aligned}
$$

(3) $y=20 x^{3}+0.002 l=0.0592$

$$
\begin{aligned}
f= & {[10.9,78.9,234.1] } \\
a^{(1)}= & {[216638.6,-1494035,-9117084,} \\
& -2354616,0.3173259 \times 10^{10},-0.2225818 \\
& \left.\times 10^{11}\right] \\
a^{(2)}= & {\left[1567328,-0.3412063 \times 10^{8},-0.9623713\right.} \\
& \times 10^{9}, 0.3600677 \times 10^{11},-0.3914506 \\
& \left.\times 10^{12}, 0.1449704 \times 10^{13}\right] \\
a^{(3)}= & -5072059,0.2541901 \times 10^{9}, 0.1173915 \\
& \times 10^{10},-0.2393105 \times 10^{12}, 0.4250562 \\
& \left.\times 10^{13},-0.2304118 \times 10^{14}\right]
\end{aligned}
$$

By inserting three normalized eigenfunctions $V_{i}(x)$ in Equations (5) and (7), steady-state deflection $z(x, t)$ of the GMF can be obtained.

\section{Propulsive modelling of boimimetic swimming}

Swimming trace of caudal fin of robot fish in liquid is as shown in Figure 6. $z(x, t)$ is the vibrating function or steady-state vibration deflection of caudal fin under excitation of alternating magnetic field.

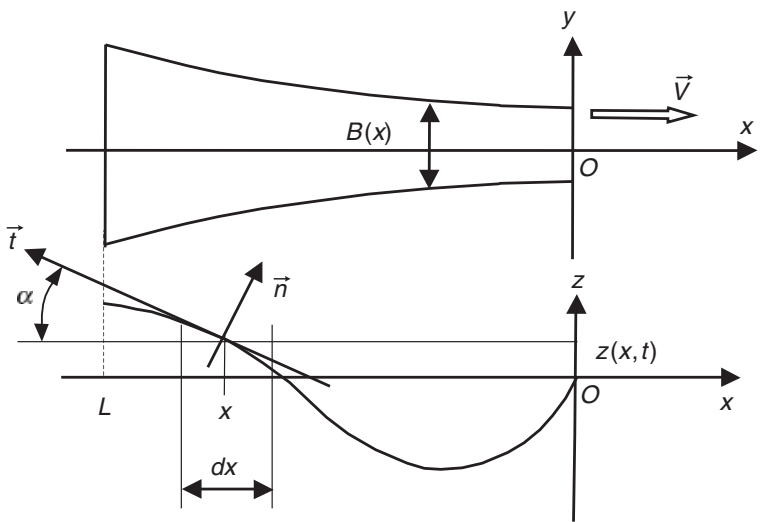

Figure 6 Motion and propulsive force analysis of the GMF fin.

The propulsive force along $x$ axis is (Zhang and Liu 2005a)

$$
\begin{aligned}
F_{x}= & -\frac{1}{2} \rho B(x)\left(\int_{0}^{l} C_{n} V_{n}\left|V_{n}\right| \tan \alpha \mathrm{d} x\right. \\
& \left.+C_{t} \int_{0}^{l} V_{t}\left|V_{t}\right| \mathrm{d} x\right),
\end{aligned}
$$

where $\rho$ is density of the fluid; $\vec{V}_{n}$, normal speed of fin surface relative to liquid; $\vec{V}_{t}$, tangent speed of fin surface relative to liquid; $C_{n}$, drag coefficient in normal direction; and $C_{t}$, drag coefficient in tangent direction.

$$
\begin{gathered}
V_{n}=-V \sin \alpha+\frac{\partial z(x, t)}{\partial t} \cos \alpha \\
V_{t}=V \cos \alpha+\frac{\partial z(x, t)}{\partial t} \sin \alpha
\end{gathered}
$$

where $V$ is the mean swimming speed or embroiled speed along axis; $\alpha$, angle between tangent of caudal fin and axis $x$. For minor vibration, $\alpha=\partial z(x, t) / \partial x$.

The calculated propulsive forces of the four caudal fins depicted in Figure 4 are shown in Figure 7. We find that the propulsion force alternates with time for each fin. When its mean value is positive, the propulsion force serves as a pushing force; and when the mean force is negative, it acts as a drag force. As a result, the robot swims forward with a slow speed if driven at the first-stage resonance frequency, and with a faster forward speed if driven at the secondstage resonance frequency. The robot swims backward if driven at the third-stage resonance frequency.

The mean propulsive force can be calculated as (Zhang and Liu 2005b)

$$
\bar{F}(f, V)=\frac{\int_{0}^{T} F_{x}(f, t) \mathrm{d} t}{T} .
$$

Drag force exerted on robot body is (Laurent and Piat 2001)

$$
F_{R}=\frac{1}{2} C_{n} \rho S_{b} V^{2},
$$




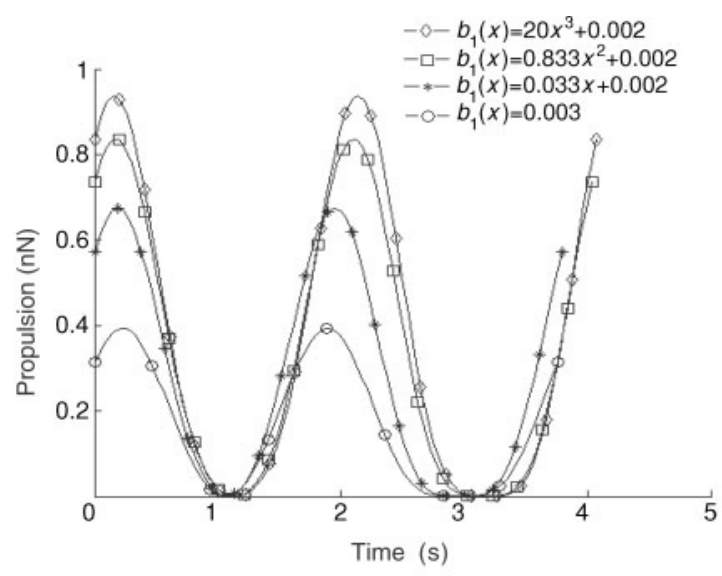

(a)

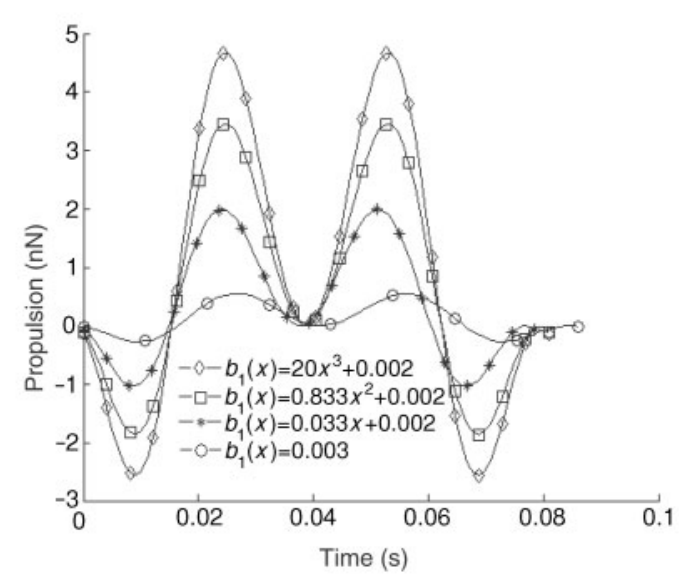

(b)

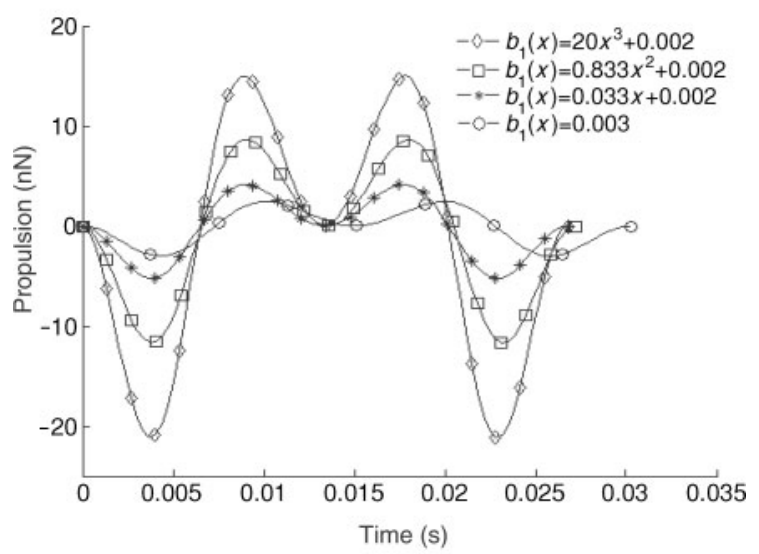

(c)

Figure 7 Propulsion of caudal fins in a cycle: (a) the first-stage resonance frequency; (b) the second-stage resonance frequency; and (c) the third-stage resonance frequency.

where $V$ is the swimming speed of robot and $S_{b}$ is the section area of robot body.

The crossing point between the mean propulsive force curve and drag force curve related to $V$ is the swimming speed (Laurent and Piat 2001). Thus, by combining the two equations of mean propulsive force and drag force, the robot swimming speed can be determined as follows:

$$
V(f)=\sqrt{\frac{2 \int_{0}^{T} F_{x}(f, t) \mathrm{d} t}{T C_{n} \rho S_{b}}} .
$$

\section{OPTIMIZATION ON SHAPE OF CAUDAL FIN}

As the solutions for the eigenfunction $V_{i}(x)$ of GMF fin are obtained by a numerical method, it is appropriate to use a discrete variate method for optimising the caudal fin configuration.

Since the most powerful propulsion and fastest swimming speed of a GMF fin actuated robot fish occur at the second-order resonance frequency (Zhang et al. 2005b), the equation $F_{x}^{(2)}\left(X^{D}\right)$ of propulsion at the second-order resonance frequency described by Equation (10) is selected as the objective function, and the problem is converted to obtain optimal configuration of caudal fin to maximize the objective function $F_{x}^{(2)}\left(X^{D}\right)$ under the constraint of constant surface area and sufficient end strength of the caudal fin.

\section{Discrete variates optimisation}

For a certain given fin surface area, smaller width of the fixed fin end makes the aspect ratio and sweepback angle larger, but weakens rigidity of the fin, resulting in less restoring force and propulsion. Therefore, the stress of GMF should not surpass its tensile strength.

Suppose a caudal fin to be optimised has the same surface area as the rectangle fin counterpart, with a surface area of $S=0.008 \mathrm{~m} \times 0.045 \mathrm{~m}$. Considering the feasibility of manufacturing the fin, the fixed fin end is selected to be no less than $4-\mathrm{mm}$ wide, that is, $b \geq 0.002 \mathrm{~m}$. For the assumed shape of caudal fin, $a \geq 0$ and $l \leq 0.09 \mathrm{~m}$, under the constraint of the given constant surface area. According to our simulation studies, when the highest power of the analytic shape function $b_{1}(x)$ increases from 1 , the largest propulsion of the caudal fin increases significantly. However, if it is higher than 9 , that is, $c>9$, the largest propulsion of the caudal fin starts to decrease obviously. Therefore, we select a reasonable range of $1 \leq c \leq 10$ for the optimisation.

Therefore, the optimisation problem is formulated as follows:

Variables need optimize, $X^{D}=\left[\begin{array}{l}a \\ b \\ c \\ l\end{array}\right]$

Objective function : $\max F_{x}^{(2)}\left(X^{D}\right)$

Constraint conditions:

$$
\begin{aligned}
& a \geq 0 \\
& 0.002 \leq b \leq 0.004 \\
& l \leq 0.09
\end{aligned}
$$




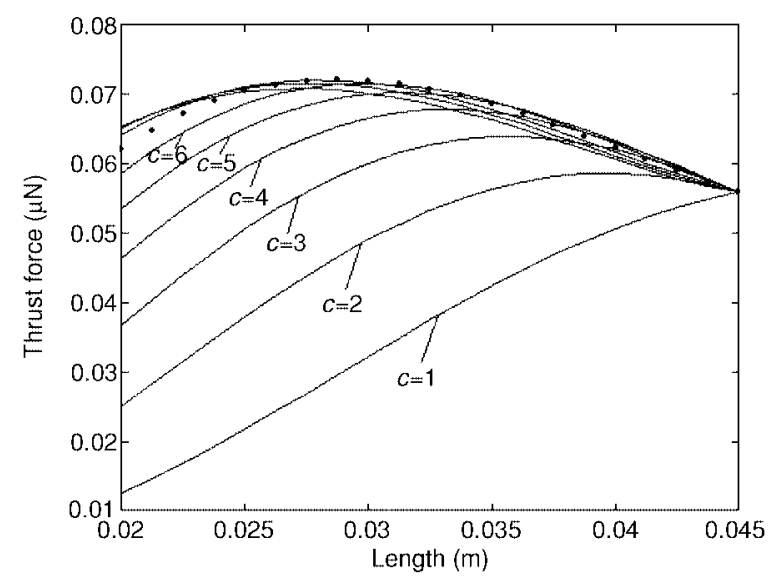

(a)

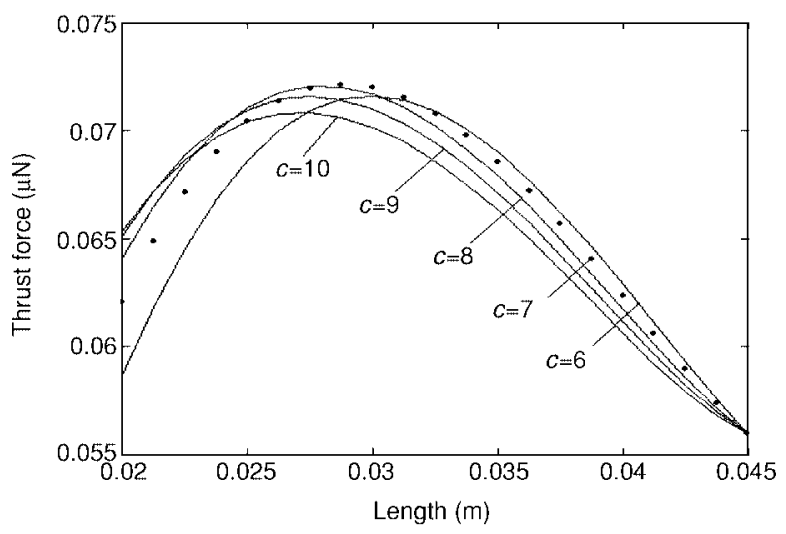

(b)

Figure 8 Propulsion diagram when $b=0.04:$ (a) $c \in[1,6]$; (b) $c \in[6,10]$.

$$
\int_{0}^{l} 2\left(a x^{c}+b\right) \mathrm{d} x=0.008 \times 0.045 .
$$

A gridding method is adopted to optimise the objective function. The parameter $b$ is divided into two sections by a step length of $0.001, b \in[0.002,0.004]$, and $c$ into nine sections by a step length of $1, c \in[1,10]$. The cases of $l$ and $a$ are discussed in the following:

(1) When $b=0.004, c$ is divided into nine sections by a step length of $1, c \in[1,10]$, and $l$ is divided into 20 sections by a step length of $0.0012, l \in[0.02,0.045]$. According to the constraint condition Equation (15), $a$ is divided into 210 dispersed points. The combination of the four parameters constitutes a four-dimensional dispersed design space, and propulsion in the design space is calculated as shown in Figure 8 , with $l$ as the $x$-axis and propulsion as the $y$-axis.

Simulation results show that the most powerful mean propulsion force in the first group is $7.2 \times 10^{-5} \mathrm{mN}$, with a fin configuration determined by the parameters $c=7$, $l=0.0284 \mathrm{~m}$ and $a=1114039400$.

(2) When $b=0.003, c$ is divided into nine sections with a step length of $1, c \in[1,10]$, and $l$ is divided into 20 sections with a step length of $0.0015, l \in[0.03,0.06]$. According to the constraint condition Equation (15), $a$ is

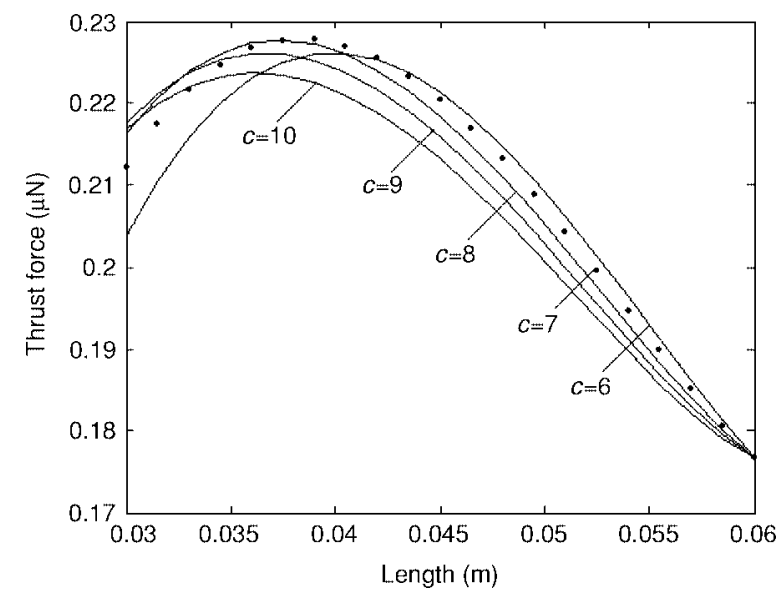

Figure 9 Propulsion diagram when $b=0.03$ and $c \in[6,10]$.

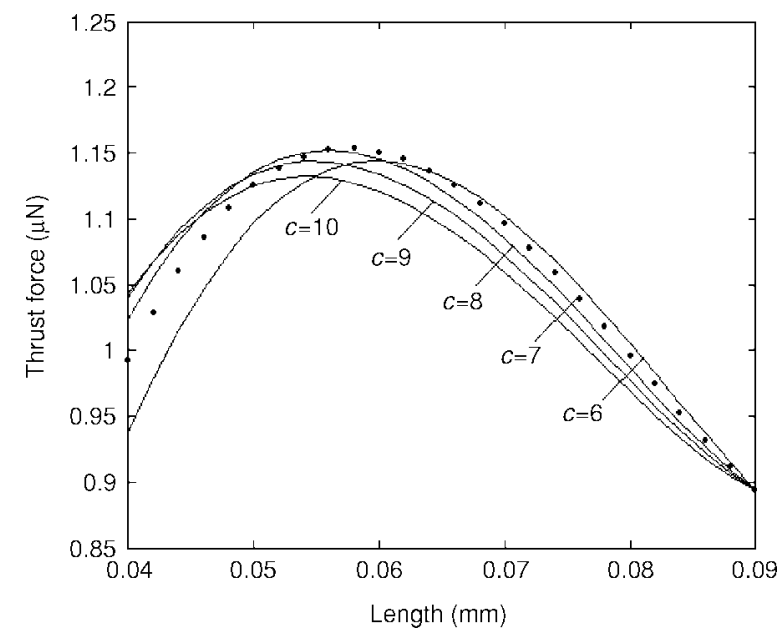

Figure 10 Propulsion diagram when $b=0.02$ and $c \in[6,10]$.

divided into 210 dispersed points. The propulsion force in this design space is calculated as shown in Figure 9. It was found in the simulation that the most powerful mean propulsion happens when $c$ is between 6 and 10. Hence, the diagram shows only the results for $c \in[6,10]$.

The largest mean propulsion force in the second group is $2.3 \times 10^{-4} \mathrm{mN}$, achieved with parameters $c=7, l=$ $0.039 \mathrm{~m}$ and $a=94170241$.

(3) When $b=0.002, c$ is divided into nine sections by a step length of $1, c \in[1,10]$, and $l$ is divided into 25 sections by a step length of $0.002, l \in[0.04,0.09]$. From Equation (15), $a$ is divided into 260 dispersed points. The propulsion force in this design space is calculated as shown in Figure 10, again for $c \in[6,10]$.

Simulation shows that the largest mean propulsion in this third group is $1.2 \times 10^{-3} \mathrm{mN}$, achieved with the parameters $c=7, l=0.058 \mathrm{~m}$ and $a=3998029.7$

(4) When $b=0.004$, the caudal fin is rectangular with a length $l$ of 0.045 under the constraint condition of equal surface area, thus providing the least propulsion with the largest mean propulsion of $7.9 \times 10^{-8} \mathrm{mN}$. 


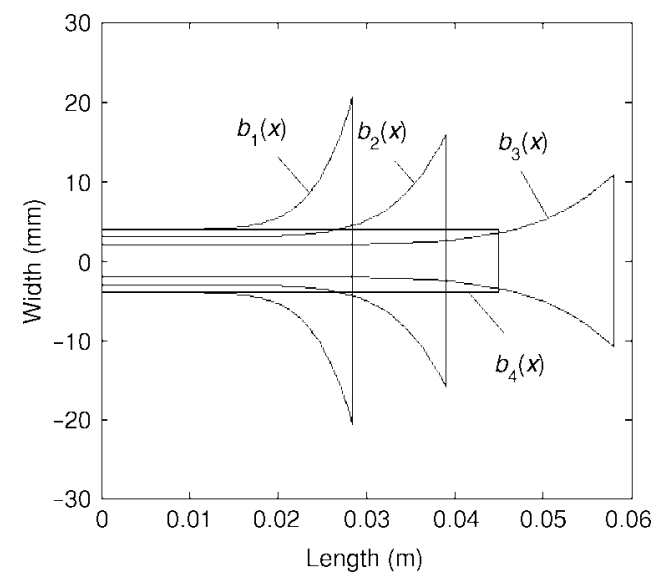

Figure 11 Optimised configuration of caudal fin.

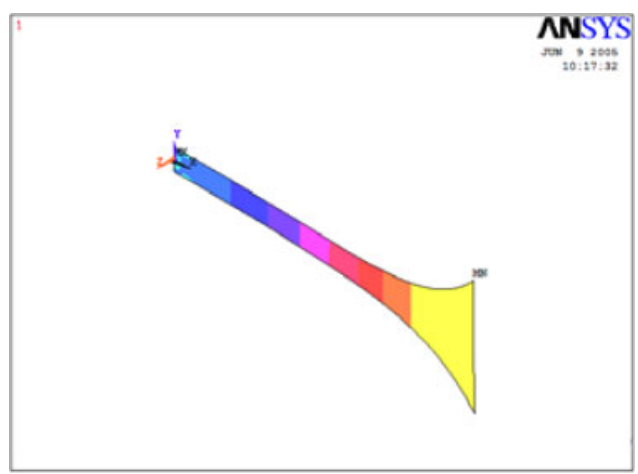

Figure 12 The contour plot of stress.

Table 2 Results of optimisation to caudal fins

\begin{tabular}{llll}
\hline$b_{\mathrm{i}}(x)=a x^{c}+b$ & $l(\mathrm{~mm})$ & $f_{2}(\mathrm{~Hz})$ & $F(\mathrm{mN})$ \\
\hline$b_{1}(x)=1.1 \times 10^{9} x^{7}+$ & 28.4 & 495 & $7.2 \times 10^{-5}$ \\
0.004 & & & \\
$b_{2}(x)=9.4 \times 10^{7} x^{7}+$ & 39 & 262 & $2.3 \times 10^{-4}$ \\
0.003 & & & \\
$b_{3}(x)=4.0 \times 10^{6} x^{7}+$ & 58 & 118 & $1.2 \times 10^{-3}$ \\
0.002 & & & \\
$b_{4}(x)=0.004$ & 45 & 228 & $7.9 \times 10^{-8}$ \\
\hline
\end{tabular}

The above simulation results are summarized in Table 2, where $f_{2}(\mathrm{~Hz})$ refers to the second-order resonance frequency and $F(\mathrm{mN})$ is the largest mean propulsion force. The four configurations of caudal fin are shown in Figure 11. By comparison, it is found that the third optimised configuration generates the most powerful propulsion, which represents the best caudal fin shape, with $b_{3}(x)=4.0 \times 10^{6} x^{7}+0.002$.

\section{Analysis on outsized caudal fin by finite element method}

Stress distribution inside an optimised caudal fin is analysed using the finite element method, and the contour plot of stress is shown in Figure 12. It is found that the highest stress of $0.11 \times 10^{7} \mathrm{~Pa}$ occurs at the end of the caudal fin, which is less than the tensile stress of polyimide, $115 \mathrm{Mpa}$,

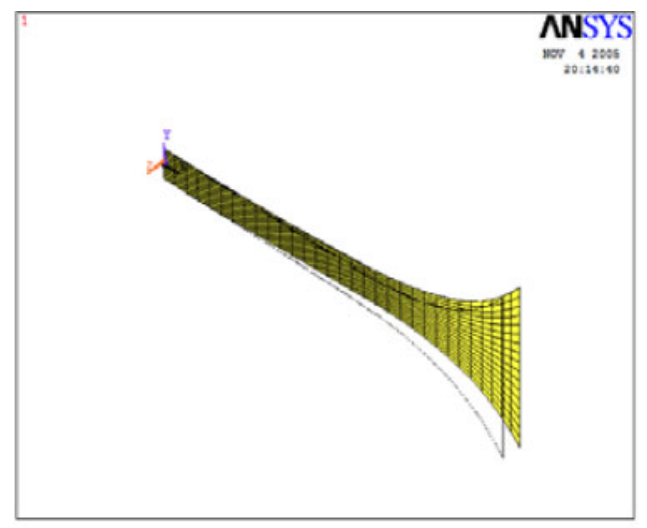

(a)

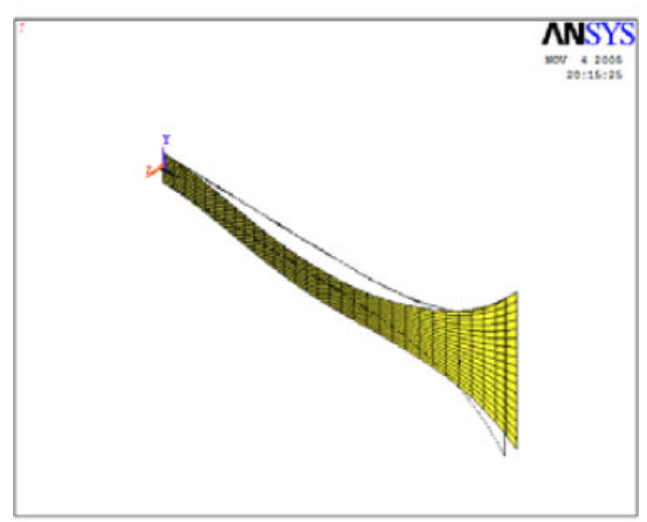

(b)

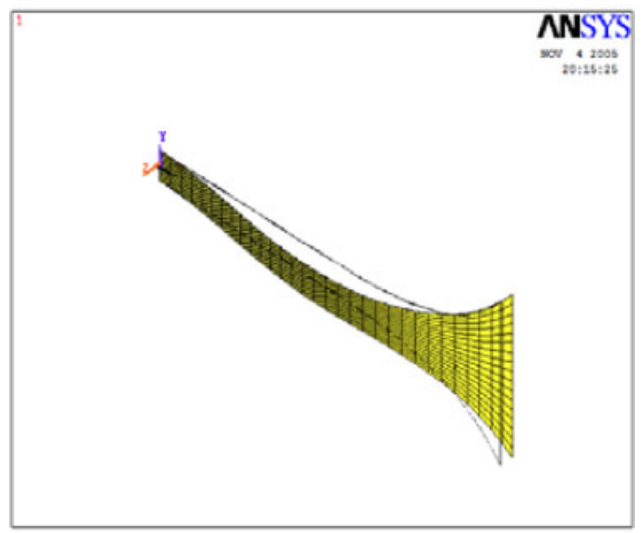

(c)

Figure 13 Deflection of three order mode shapes: (a) mode shape in first order; (b) mode shape in second order; and (c) mode shape in third order.

and the tensile stress of giant magnetostrictive material, $28 \mathrm{Mpa}$.

Finite element analysis on vibrating deflection of the first three mode shapes of the optimised caudal fin is also conducted, and the results are as shown Figure 13. According to the simulated deflection diagram of the first three mode shapes and relationship between propulsion and mode shape, it is obvious that the first-order mode shape vibrates in swings, resulting in low propulsion 


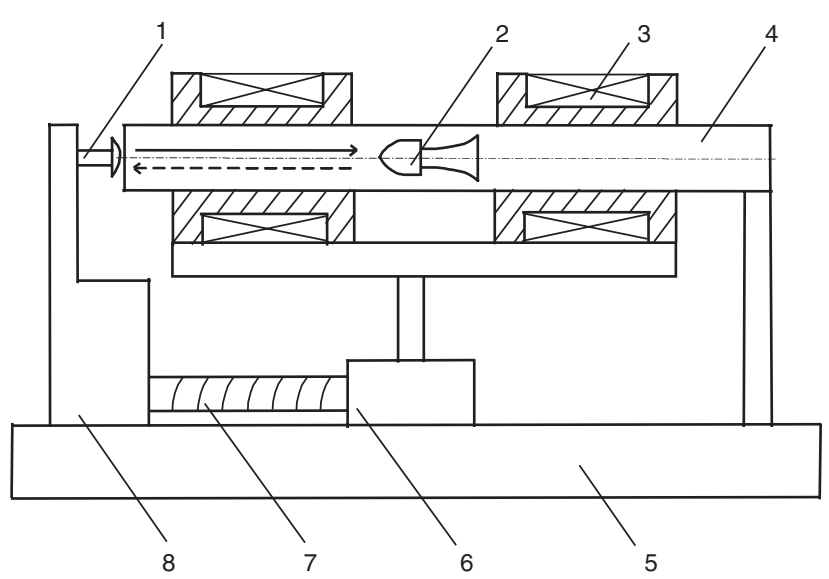

Figure 14 The experimental set-up for robot fish: 1 , ultrasonic sensor; 2 , robot fish; 3 , magnetic coils; 4 , pipe; 5 , table; 6 , step motor; 7 , screw; and 8 , voltmeter.

Table 3 Fin shapes for verification experiments

\begin{tabular}{llll}
\hline$b_{\mathrm{i}}(x)=a x^{c}+b$ & $l(\mathrm{~mm})$ & $f_{2}(\mathrm{~Hz})$ & $F(\mathrm{mN})$ \\
\hline$b_{1}(x)=4.0 \times 10^{6} x^{7}+0.002$ & 58 & 118 & $1.2 \times 10^{-3}$ \\
$b_{2}(x)=7.2 \times 10^{5} x^{6}+0.002$ & 60 & 156 & $8.3 \times 10^{-4}$ \\
$b_{3}(x)=0.004$ & 45 & 228 & $7.9 \times 10^{-8}$ \\
\hline
\end{tabular}

and drive efficiency, whereas the mode shape in second order vibrates in undulated motion with single inflexion point as fish do, resulting in high propulsion force and drive efficiency. The mode shape in third order vibrates in undulated motion with double inflexion point as fish do, resulting in reverse swimming of robot fish.

\section{SIMULATION ON SWIMMING SPEED AND ITS VERIFICATION}

On the basis of the optimisation and analysis results in the above sections, three types of fins in different configurations are selected for comparison, as listed in Table 3 , where $b_{1}(x)$ is the optimised fin shape; $b_{2}(x)$ is a selected fin shape with a power of six; and $b_{3}(x)$ is the rectangle fin shape for reference. All three fins have the same surface area.

From Equation (11), the mean swimming speed for each type of selected fins is calculated with respect to driving frequency. The simulation results are shown in Figure 15, along with experimental results.

The selected fins are experimentally tested. The experimental set-up includes a magnetic field controller, double-coil magnetic excitation system (Zhang et al. 2004), an ultrasonic sensor system (Zhang et al. 2005a) and a fish robot, as illustrated in Figure 14. The pipe segment filled with liquid has a diameter of $40 \mathrm{~mm}$ and a length of $1500 \mathrm{~mm}$.

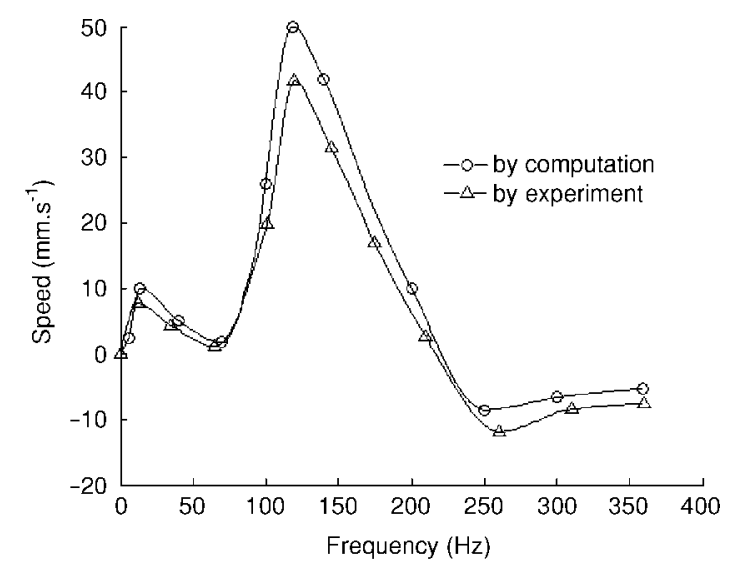

(a)

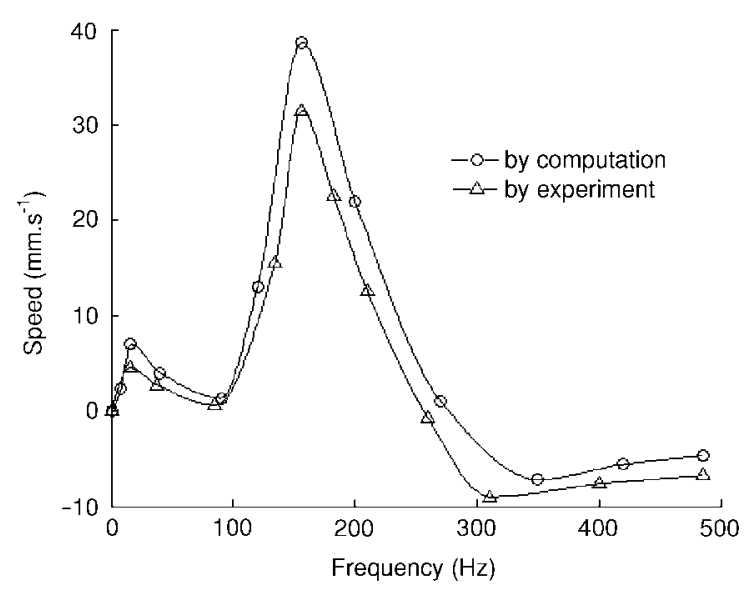

(b)

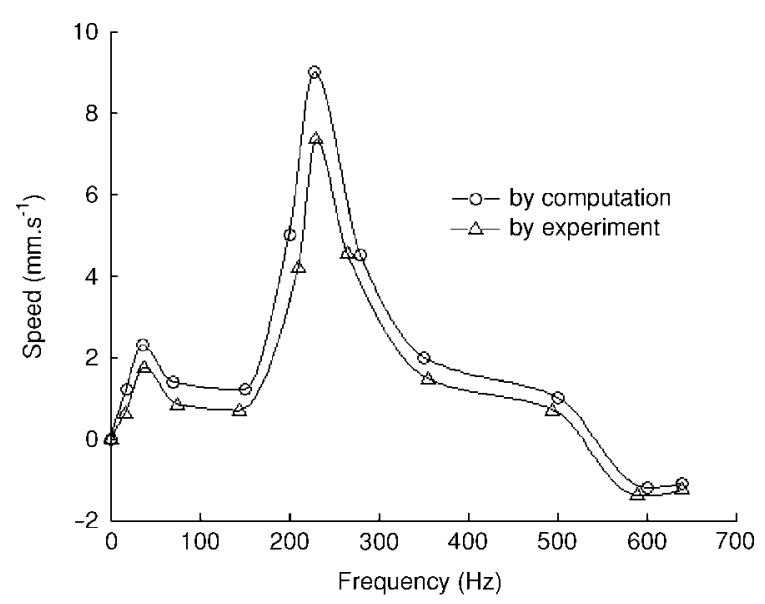

(c)

Figure 15 Simulation and experiments on swimming speeds of robot fish with different shape of caudal fin: (a) $b_{1}(x)=$ $4.0 \times 10^{6} x^{7}+0.002 ;$ (b) $b_{2}(x)=7.2 \times 10^{5} x^{6}+0.002 ;$ and (c) $b_{3}(x)=0.004$.

For all the three fish robots with three types of fin shape, the magnetic field excitation current is set at $0.25 \mathrm{~A}$, and the driving frequency is changed from 0 to $650 \mathrm{~Hz}$. The mean swimming speed of each fish robot is measured by the ultrasonic sensor system, and the results are plotted in Figure 15. 
The experimental results are summarized as follows:

- Fish robot swims forward at small mean swimming speed with large sway when driven near the first-order resonance frequency, and robot swims forward at a maximum swimming speed and in good stability when driven at the second-order resonance frequency. The fish robots swim backward at small swimming speed when driven nearby the third-order resonance frequency. The results are in coincidence with previous theoretical analysis and simulation results using the finite element method.

- For the three selected fin shapes, their mean swimming speed varies significantly. The fish robot with the optimised fin swims at a much higher maximum speed compared with its rectangular counterpart. The results have demonstrated that fin shape does have great influence on swimming speed and the correctness of the optimised result.

\section{CONCLUSIONS}

In this work, a micro fish robot with optimised GMF caudal fin is developed and studied analytically and experimentally. The force oscillation dynamic model of a GMF cantilever with variable cross-sectional area is derived, and the propulsive model of the fish robot in liquid is established. By dispersing four-dimensional continuous space variables for describing the shape of caudal fin using a gridding method, dispersed four-dimensional space variables are obtained. Using the propulsion at the second-order resonance frequency as the objective function, the caudal fin shape is optimised under the constraint of constant fin area and sufficient strength of the fixed fin end. Fish robots with fins of several representative shapes are analysed and experimentally tested. Both theoretical analysis and experimental results show the effectiveness of the proposed caudal fin optimisation approach using the discrete variate method.

Further research work is planned as follows:

(1) Optimisation of bi-directional model. That is, to make the largest forward swimming speed to be the same as that of backward swimming speed with high efficiency.

(2) Minimization of the physical size of the fish robot. The goal is to reduce the robot dimensions to millimetre level, which is going to be a big challenge and requires study of dimensional effect and new propulsive force theory based on resonance frequency.
(3) Research on close-loop control of bi-directional swimming.

\section{ACKNOWLEDGMENTS}

This work was supported in part by the National Natural Science Foundation of China through grant \#60275034 and in part by the Natural Science Foundation of Liaoning Province through grant \#20032119.

\section{REFERENCES}

Body C, Reyne G, Meunier G. 1997. Nonlinear finite element modeling of magneto-mechanical phenomenon in giant magnetostrictive thin films, IEEE Trans Magnet, 33(2), 1620-3.

Breder CM. 1926. The locomotion of fishes. Zoologica, 4, 159-256.

Honda T, Arai KI, Yamaguchi M. 1997. Basic properties of magnetostrictive actuators using $\mathrm{Tb}-\mathrm{Fe}$ thin film. IEICE Trans Electron, E80-C(2), 232-7.

Laurent G, Piat E. 2001. Efficiency of swimming microrobots using ionic polymer metal composite actuators. In Proceedings of the IEEE International Conference on Robotics \& Automation, 2001, Seoul, Korea, p. 3914-9.

Quant E, Seemann K. 1995. Fabrication of giant magnetostrictive thin film actuators. In Proc IEEE MEMS'95, Amsterdam, 1995, p. 273-7.

Sfakiotakis M, Lane DM, Bruce J, et al. 1999. Review of fish swimming modes for aquatic locomotion. IEEE $\mathcal{7}$ Ocean Eng, 24(2), 237-52.

Tse F, Morse IE, Hinkle RT. 1978. Mechanical vibration theory and application, 2nd ed. Boston: Allyn and Bacon Inc.

Webb PW, Weihs D. 1983. Fish Biomechanic. New York: Praeger.

Zhang Y, Liu G. 2005. Design, analysis and experiments of a wireless swimming micro robot. In Proceedings of the IEEE International Conference on Mechatronics \& Automation, Niagara Falls, Ontario, Canada, 2005, p. 946-51.

Zhang Y, Liu G. 2005. Wireless micro biomimetic swimming robot based on giant magnetostrictive thin films. In Proceedings of the IEEE International Conference on Robotics \& Biomimetics, Hong Kong and Macau, 2005, p. 195-200.

Zhang Y, Guo R, Liu Y. 2004. On-line locating method on micro swimming robot inside pipe. $\mathcal{F}$ Harbin Inst Technol, 36(10), 1382-4.

Zhang Y, Liu W, Guo R. 2005a. Research on driving magnetic field system for a wireless micro swimming robot. 7 Chin Assoc Automat, Robot. 27(2), 63-67.

Zhang Y, Liu W, Jia Z. 2005b. Biomimetic swimming properties of a wireless micro robot driven by outside magnetic field. Chin $\mathcal{F}$ Mech Eng, 41(10), 51-6. 

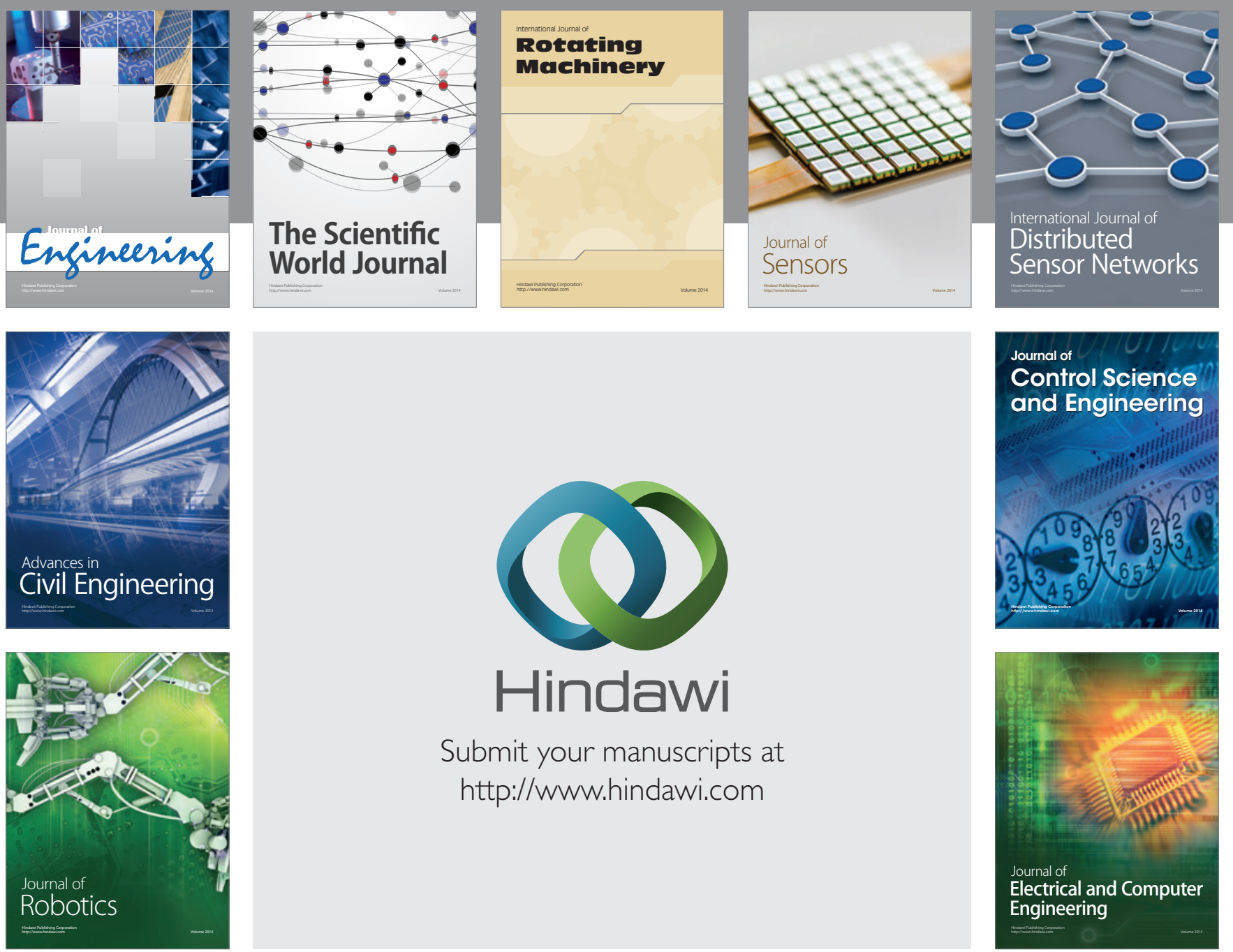

Submit your manuscripts at

http://www.hindawi.com
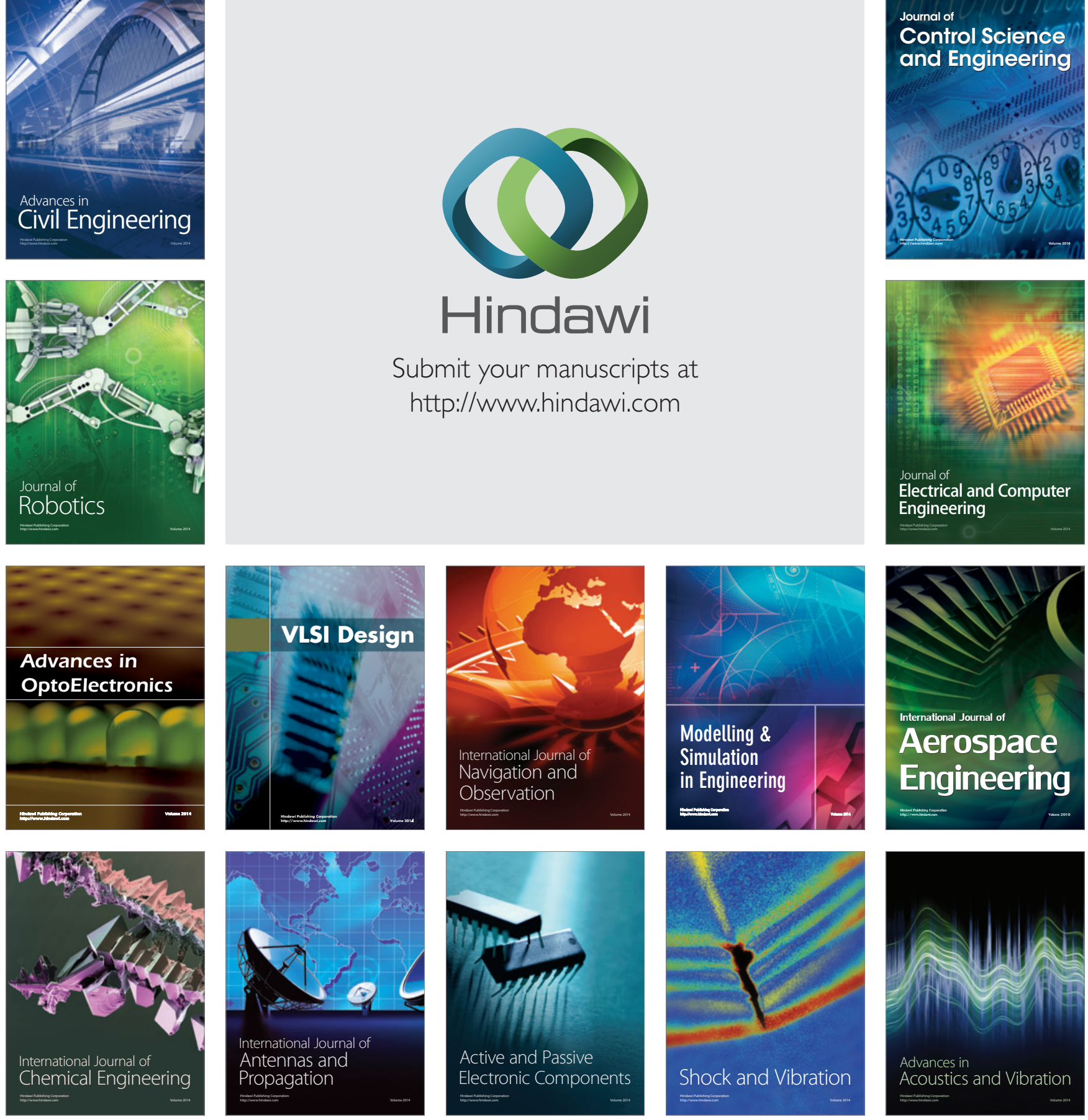